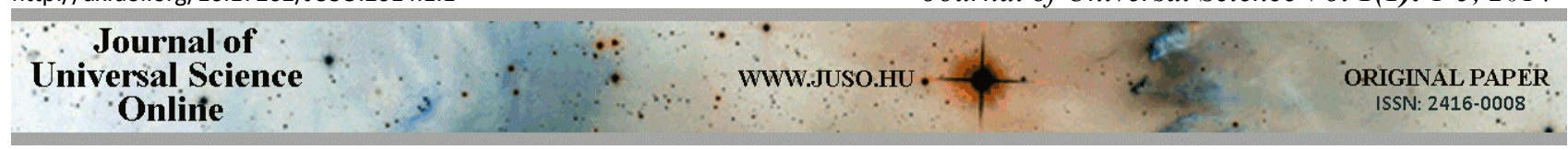

\title{
Some basic mathematical features of the simultaneous convection anomalous diffusion transport processes
}

\author{
Ágnes BÁLINT ${ }^{1}{ }^{* *}$ and Csaba MÉSZÁROS ${ }^{2}$
}

${ }^{1}$ Institute of Environmental Engineering, Óbuda University, Doberdó u. 6., Budapest, H-1034 Hungary
${ }^{2}$ Department of Physics and Process Control, Szent István University, Páter K.u.1., Gödöllö, H-2103 Hungary

${ }^{*}$ Corresponding Author; e-mail: balint.agnes@rkk.uni-obuda.hu

Received: 26 September 2014 / Revised: 15 October 2014 / Accepted: 15 December 2014

Keywords: Convection, Anomalous Diffusion, Percolative-Fractal systems

\begin{abstract}
The basic mathematical features of the anomalous diffusion processes are discussed in detail, together with some of the open research subdomains belonging to it. Due to its practical importance in engineering applications, the possibilities of the necessary mathematical refinements are proposed and justified for the general case of the simultaneous convectionanomalous diffusion processes taking place through porous bulk materials.
\end{abstract}

\section{Introduction}

According to the most general descriptions of the topic, the transport processes can most correctly be treated within framework of the extended irreversible thermodynamics [5]. The transport phenomena occuring in soils represent no exception. It is well-known in the contemporary literature of soil transport processes e.g. [3], that modelling of these processes is based frequently on the application of the classical advectiondispersion equation (ADE), i.e. on of the most important equations of the physical kinetics, written here in three - dimensional form:

$$
\frac{\partial c}{\partial t}=-\vec{v} \cdot \nabla c+D \nabla^{2} c, c=c(\vec{r}, t)
$$

where $c=c(\vec{r}, t)$ is the resident fluid concentration, $t$ denotes time, $r$ is distance, and $D$ is the hydrodynamic dispersion coefficient. This equation is even today a very useful tool at modelling of data emanating from serious soil experiments e.g. those controlled on the base of the combined the mobile-immobile model with an asymptotic scale-dependent dispersivity models [4]. However, equation (1) is not always enough to give an accurate description of diffusion processes taking place in soils, because of their genuine mesoscopic structure. The socalled non-classical diffusion- (characterizing for instance dispersion of pollutants in rivers and estuaries - a phenomenon known under the name of Taylor-dispersion [5]) and anomalous diffusion phenomena, which may appear in the case of stochastic motion of particles with non-Brownian character are good illustrations e.g. [8]. All these digressions from the ordinary diffusion are also called transport processes of non-Fickian character and call for serious further advances 


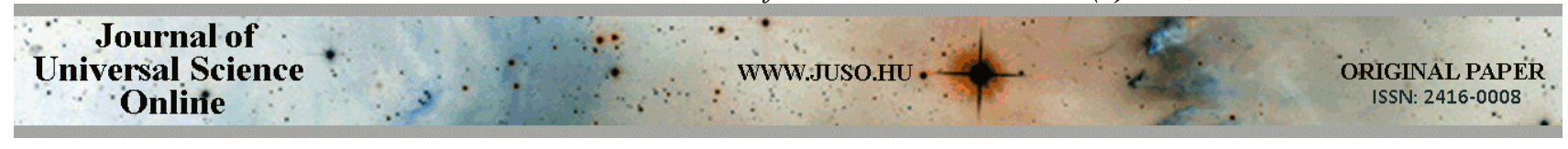

in the theory of stochastic processes. In the forthcoming sections we will describe briefly and justify application of a powerful general formalism able to reflect nonlocal character

\section{Material and methods}

It is well-known nowadays, that investigation of both percolation phenomena [9] and systems with fractal character [10] belong to the rapidly advancing frontiers of contemporary mathematical- and statistical physics with numerous open research subdomains, whose investigation represents a very fruitful research topic in future. There are also numerous systems exhibiting both of the two above-mentioned features, and are usually called percolative-fractal (PF) systems. Among them, investigation the simultaneous convection - anomalous diffusion processes may also represent a novel-type research domain of crucial importance in this area [7]. Accordingly, we

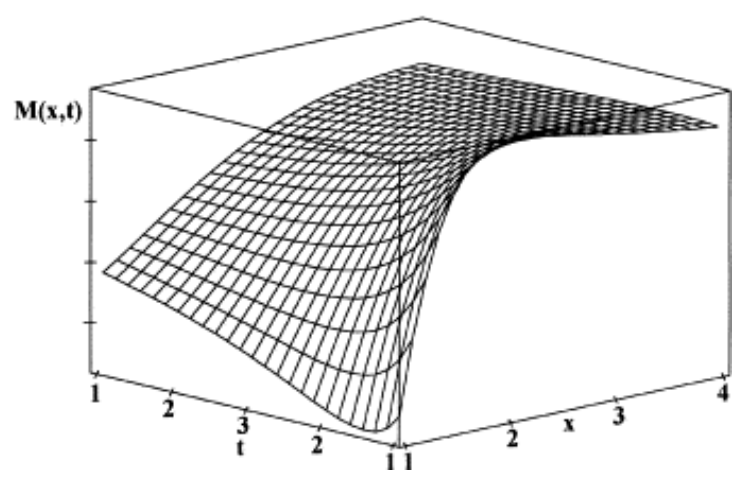

(a) and possible „memory” effects characterizing the genuine transport processes in the systems having percolative-fractal character in mesoscopic sense. think, that only the common application of the powerful calculation methods emanating from non-equilibrium thermodynamics and percolation theory may provide a genuine background for accurate modelling of (in general case:) coupled transport processes taking place in porous media. For instance, this modelling strategy has been applied rather rarely in one of the most obvious research fields of the topic - in the theory of drying processes [6]. (It was taken into account in this previous study of ours, that the diffusion coefficient depends on difference of the actual (p) and critical percolation probability $\left(\mathrm{p}_{\mathrm{c}}\right)$ in agreement with the relevant scaling relationship as

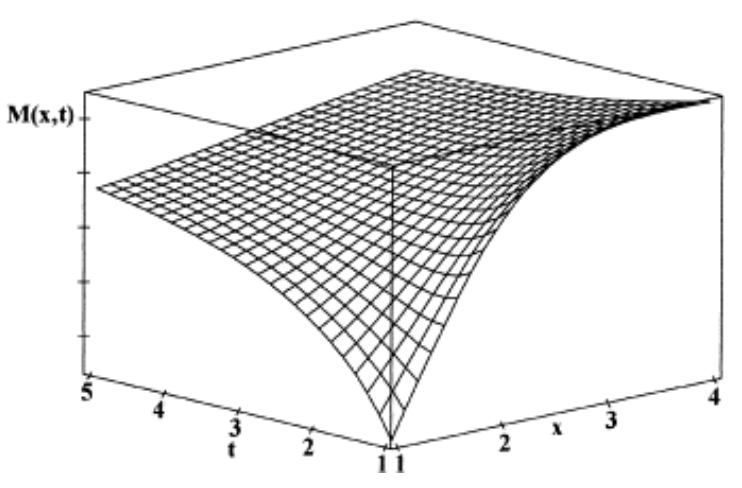

(b)

Fig. 1. Scaling behaviour of the moisture level distribution in the case of coupled heat and mass transfer in porous medium (in relative units) for (a) $\Delta \mathrm{p}=0,001$, (b) $\Delta \mathrm{p}=0,002$ values of the percolation probability difference after [6].

$\mathrm{D} \propto(\Delta \mathrm{p})^{\mathrm{t}_{\mathrm{p}}}, \Delta \mathrm{p}=\mathrm{p}-\mathrm{p}_{\mathrm{c}},(\mathrm{t} \in[1.1,1.6]) \quad$ and then, an a posteriori refinement of the basic analytical solution of the general coupled system of parabolic-type partial differential equations was realized.) Since soil columns represent an archetypal case of PF systems, their investigation from the point of view of newest transport theories (see e.g. [4]) may result in more refined descriptions of transport processes taking place in them, as well as in predicting of new types of diffusion phenomena. According to the key concept of this theory, the density distribution function $\rho(x, t)$ (of the moisture content of the matter, 


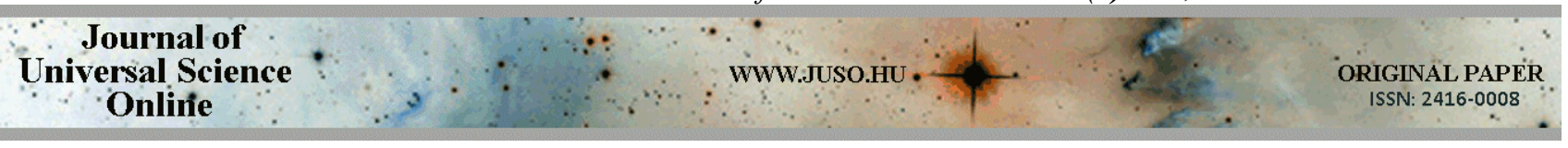

for example) obeys the following scaling

$$
\rho(x, t)=t^{-\mu} \cdot q\left(x t^{-\mu}\right),
$$

where $\mu$ is the scaling exponent, while $q(x, t)$ is a time-, and space-dependent distribution function, which is not necessarily of Gaussian-type. If $\mu>1 / 2$ we speak about superdiffusion, while for $\mu<1 / 2$ the so-called subdiffusive processes are taking place in the relationship of general character:

system. In such transport processes the usual normal (i.e. Gaussian) form of the diffusion packet is not necessarily maintained and therefore cannot be modelled without additional supplementary relations, explained here by crucial scaling relations from the contemporary statistical physics. Accordingly, the transport equation can be written as:

$$
\frac{\partial^{\omega} \rho(x, t)}{\partial t^{\omega}}=D K(x ; \rho(x, t))+\frac{t^{-\omega}}{\Gamma(1-\omega)} \delta(x),
$$

where $D$ is the diffusion coefficient having positive constant value, $\omega$ is an exponent in the probability distribution formula relevant for asymptotic solution of the Montroll-Weiss problem [10] with possible values of $\omega<1$. The fractional partial derivative with respect to time is explained by use of the RiemannLiouville operators, i.e.:

$$
\frac{\partial^{\omega} c(x, t)}{\partial t^{\omega}}=\frac{1}{\Gamma(1-\omega)} \frac{\partial}{\partial t} \int_{0}^{t} \frac{c(x, \tau)}{(1-\tau)^{\omega}} d \tau,
$$

where the symbol $\Gamma$ as usual, denotes the Gamma-function and $\delta$ is Dirac's delta distribution. The operator expression $K(x ; \rho(x, t))$ corresponding to the diffusion term on the right-hand side of (4) indicates general character of its, formally explained by a variety of different mathematical expressions (to be discussed below).

\section{Results and discussions}

In order to set up a realistic useful new mathematical model for describing moisture transfer in soils characterized by diffusion processes with non-local character and
By taking the fractional partial derivative of the order of $1-\omega$ of both sides of (3), and generalizing the transport equations to manydimensional case, we get:

$$
\frac{\partial \rho}{\partial \mathrm{t}}=\mathrm{D} \cdot \Delta^{\alpha / 2} \frac{\partial^{1-\omega} \rho(\overrightarrow{\mathrm{r}}, \mathrm{t})}{\partial \mathrm{t}^{1-\omega}}+\delta(\overrightarrow{\mathrm{r}}) \delta(\mathrm{t})
$$

where product of the Dirac's deltadistributions on the right-hand side describes the instantaneous initial - boundary conditions. In these equation $\alpha=1 / \mu$ (and takes its possible values from the semi-closed interval $(0,2])$ and represents the characteristic exponent relevant for description of stable transport processes in PF systems. Obviously, taking the value $\alpha=2$ (at $\omega=0$ ) will directly return the classical diffusion equation. The fractional partial derivatives with respect to space coordinates can also be explained by Riemann-Liouville operators $[1,10]$.

convection, we combine here the abovediscussed equations (1) and (5). Therefore, we propose here application of the following new-type transport equation: 


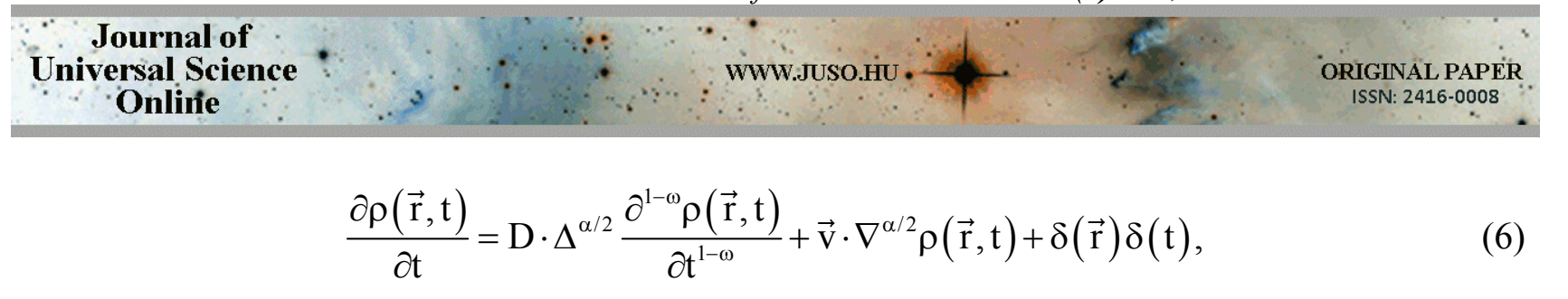

where a generalization of the term describing convective motions is performed. This extension of the classical formalism is realized by introducing fractional gradient and Laplacian operators, instead of the usual ones. This extension can be described symbolically as:

$$
\Delta=\nabla^{2} \equiv \nabla \cdot \nabla \rightarrow \Delta^{\alpha / 2}=\left(\nabla^{2}\right)^{\alpha / 2} \equiv\left(\nabla^{\alpha / 2}\right)^{2} \Rightarrow \nabla \rightarrow \nabla^{\alpha / 2}
$$

According to our knowledge, the formula (6) has not been applied in the theory of transport processes linked to anomalous diffusion, because they have not been connected so strongly to the scaling relations as it is done here. Formally, this can also be seen from some very serious earlier studies, e.g. [2] where smaller number of fractional derivation indices are used.

\section{Conclusions}

In the present work a new generalization of the classical advection - diffusion formula is proposed on base of the contemporary stochastic theory of anomalous diffusion processes. Since the relevant theoretical foundations are based on the self-similarity principle, the whole formalism is directly connected to the most confident scaling methods of statistical physics. The formalism proposed may lead to further new modelling research methods for describing anomalous transport phenomena in soils.

\section{Acknowledgements}

The authors acknowledge support of the MÖB-DAAD Project (No. 55731).

\section{References}

1. Atanacković, T., Pilipović, S., Stanković, B., Zorica, D.: (2014) Fractional Calculus with Applications in Mechanics: Vibrations and Diffusion Processes (ISTE); London: Wiley \& Sons. Inc.

2. Chechkin, A.V., Klafter, J., Sokolov, I.M.: (1992)

Fractional Fokker - Planck equation for utraslow kinetics. http://arxiv.org/pdf/cond-mat/0301487

3. Cortis, A., Berkowitz, B.: (2004) Anomalous Transport in „Classical” Soil and Sand Columns. Soil Science Society America Journal 68: pp 1539 -1548 .
4. Gao, G.Y., Zhan, H.B., Feng, S.Y. Fu, B.J. Huang, G. H. (2012) A mobile-immobile model with an asymptotic scale-dependent dispersion function. Journal of Hydrology 424: pp. $172-183$.

5. Jou, D., Casas-Vazquez, J., Lebon, G.: (2001) Extended irreversible thermo-dynamics ( ${ }^{\text {rd }}$ Ed.), Berlin - Heidelberg - New York: Springer - Verlag

6. Mészáros, Cs., Farkas, I., Bálint, Á.: (2001) A new application of percolation theory for coupled transport phenomena through porous media. Mathematics and Computers in Simulation 56: pp. 395-404. 


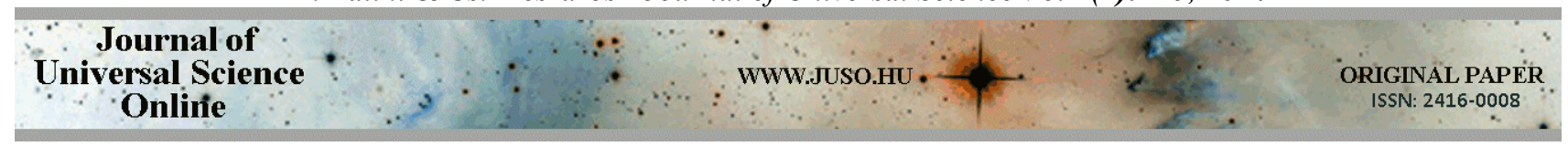

7. Mészáros, Cs., Farkas, I., Gottschalk, K., Földi, A., Bálint, Á.: Novel-type solutions of the convection anomalous diffusion equation for porous media. $19^{\text {th }}$ International Drying Symposium (IDS 2014) Lyon, France, August 24-27, 2014.

8. Metzler, R., Klafter, J.: (2003) The random walk's guide to anomalous diffusion: a fractional dynamics approach. Physics Reports - Review Section of Physics Letters 339:(1) pp. $1-77$.
9. Stauffer, D., Aharony, A.: (1994) Introduction to Percolation Theory $\left(2^{\text {nd }} d\right.$.), London: Taylor \& Francis

10. Uchaikin, V.V.: (2003) Anomalous self-similar diffusion and Lévy-stable laws. Physics - Uspekhi 46:(8) pp. $821-849$. 